\title{
Epidemiology and clinical study of pediatric psoriasis on black skin in Dakar, Senegal
}

\author{
Ndiaye Maodo ${ }^{1}$, Dioussé Pauline ${ }^{3}$, Diallo Moussa ${ }^{1}$, Diop Assane ${ }^{2}$, Diatta Boubacar Ahy ${ }^{1}$, Niang \\ Suzanne Oumou ${ }^{1}$, Diallo Salimatou ${ }^{1}$, Seck Ndeye Bougoul ${ }^{1}$, Diadie Saer ${ }^{1}$, Ndiaye Mame Téné ${ }^{\text {, }}$ \\ Ly Fatimata ${ }^{2}$, Dieng Mame Thierno ${ }^{1}$, Kane Assane ${ }^{1}$ \\ ${ }^{1}$ Service Dermatologie hôpital Aristide Le Dantec BP: 3001 \\ ${ }^{2}$ Institut d'hygiène sociale \\ ${ }^{3}$ Service Dermatologie de l'hôpital de Thiès
}

\section{Email address:}

maodo7ndiaye@yahoo.fr (N. Maodo)

\section{To cite this article:}

Ndiaye Maodo, Dioussé Pauline, Diallo Moussa, Diop Assane, Diatta Boubacar Ahy, Niang Suzanne Oumou, Diallo Salimatou, Seck Ndeye Bougoul, Diadie Saer, Ndiaye Mame Téné, Ly Fatimata, Dieng Mame Thierno, Kane Assane. Epidemiology and Clinical Study of Pediatric Psoriasis on Black Skin in Dakar, Senegal, Science Journal of Clinical Medicine. Vol. 2, No. 2, 2013, pp. 43-46.

doi: $10.11648 /$ j.sjcm.20130202.12

\begin{abstract}
Introduction: In subsaharian African countries there are so few data concerning the psoriasis of the child. Our objective was to determine the epidemiological, clinical and therapeutic aspects of the pediatric psoriasis in a dermatology unit, at Dakar in Senegal. Patients and methods: This was an 8 years (2004-2011) retrospective study conducted at the Dermatological clinic of HALD. All patients under 16 years old with psoriasis were included. Results: We report 40 cases of psoriasis of the child. The sex ratio was 1.05 and the average age of 6 years. The atopic status was found in 4 cases as follows: 3 cases of asthma and 1 case of atopic dermatitis. The average time of consultation was 5 months. The way the patients consulted was: 28 cases consulted a general practitioner, 6 cases seen by a paramedic and 5 patients consulted a traditional healer. The pruritus was present in 14 patients. The psoriasis vulgaris was the predominant form with 20 cases. The other forms were: 12 cases of guttate psoriasis, 6 cases of psoriasis universalis, 1 case in pustular and 1 case erythrodermic. The topical glucocorticoids were prescribed in all the patients. The recurrence was found in 5 cases and 8 patients were lost of follow up. Conclusion: Psoriasis is a rare and benign condition in children with predominance of simple forms such as psoriasis vulgaris.
\end{abstract}

Keywords: Psoriasis, Children, Dakar

\section{Introduction}

Psoriasis is a chronic inflammatory disease characterized by an acceleration of epidermal turnover manifested by erythematous and scaly lesions, sometimes pustules and extracutaneous signs including articular [1]. Data on pediatric psoriasis are rare in sub-Saharan Africa [2]. Our objective was to determine the epidemiological, clinical and therapeutic characteristics of pediatric psoriasis on black skin.

\section{Patients and Methods}

This was a retrospective study with a duration of eight years (2004-2011), performed in the Department of Dermatology of the Hospital Aristide Le Dantec (HALD). Were included all patients with psoriasis and under the age of 16 years. The diagnosis of psoriasis was clinical and histological.

\section{Results}

We report 40 cases of pediatric psoriasis during this period of eight years. Pediatric psoriasis accounted for $0.5 \%$ of children seen in consultation and $19.4 \%$ of psoriatic patients. The annual incidence was 4.8 on average. Sex ratio was 1.05 and the average age was 06 years, ranging from 3 months to 16 years. Age ranges [0-4] and [5-9] were the most represented in our series (Table I). Atopy was observed in 4 cases, as asthma in 3 cases and atopic dermatitis in 1 case. The average time of consultation was 5 months with extremes ranging from 1 month to 3 years. 
Previous support for consultation before the dermatologist was done by the family doctor in 28 cases, a paramedic in 6 cases and a traditional healer in 5 cases. Established treatments were: Penicillin G in 3 cases, antifungals in 5 cases, topical corticosteroids in 6 cases and African herbal medicines in 5 cases. Pruritus was noted in 14 cases. Identified clinical forms of psoriasis were: vulgaris in 20 cases (Figure 1), guttata in 12 cases, universalis in 06 cases (Figure 2), pustular and erythrodermic (Figure 3) in each one case. Various locations were identified in 21 patients: folds in 8 cases (Figure 4), palmoplantar in 7 cases (Figure 5), the scalp in 4 cases (Figure 6), diapers in 2 cases and mucosal in the form of geographic tongue. The rest of the examination highlighted a streptococcal infection in 5 patients; pharyngeal localization in 3 cases and 2 cases of otitis; in children presenting guttata form. The hemogram showed an neutrophilic leukocytosis in 5 patients. A skin biopsy performed in all patients was contributory to the diagnosis of psoriasis. Retroviral HIV serology was negative. Treatments were topical corticosteroids in 33 cases, emollients in 22 cases, calcipotriol in 8 cases and antistreptococcal antibiotics in 5 cases. After 2 months of treatment, a positive evolution was noted in 25 cases, marked by complete regression of lesions. Recurrent disease was observed in 5 cases and 08 patients were lost to follow.

Table 1. distribution of patients according to age groups.

\begin{tabular}{lll}
\hline Age group & Headcount & Pourcentage \\
\hline [0-4 years] & 16 & $40 \%$ \\
[5-9 years] & 14 & $35 \%$ \\
[10-14 years] & 7 & $17.5 \%$ \\
[15-16 years] & 3 & $7.5 \%$ \\
\hline
\end{tabular}

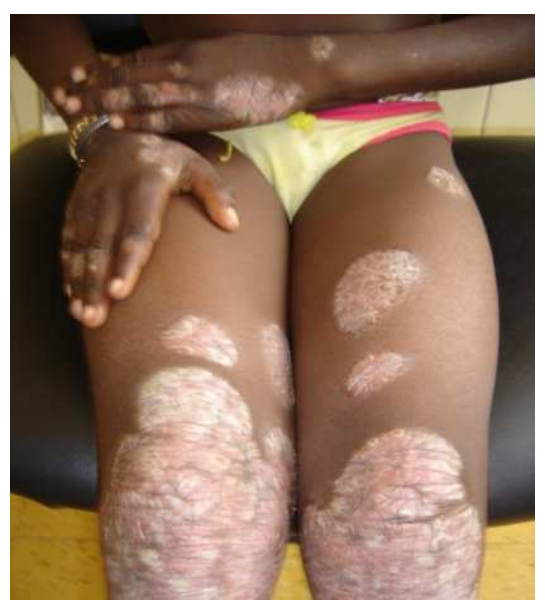

Figure 1. psoriasis vulgaris.

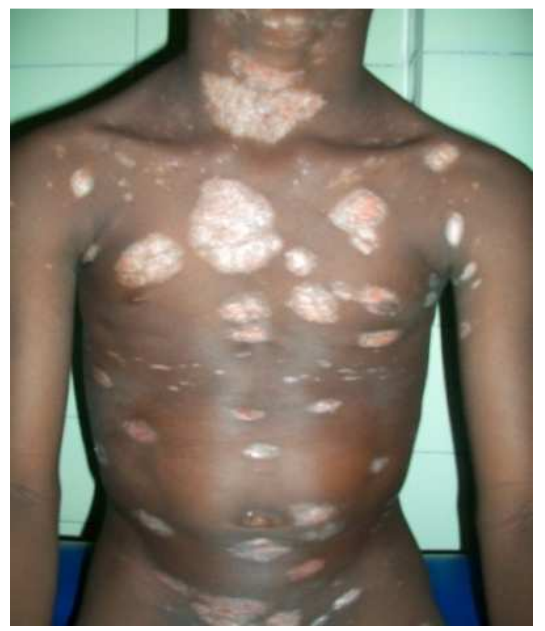

Figure 2. psoriasis universalis.

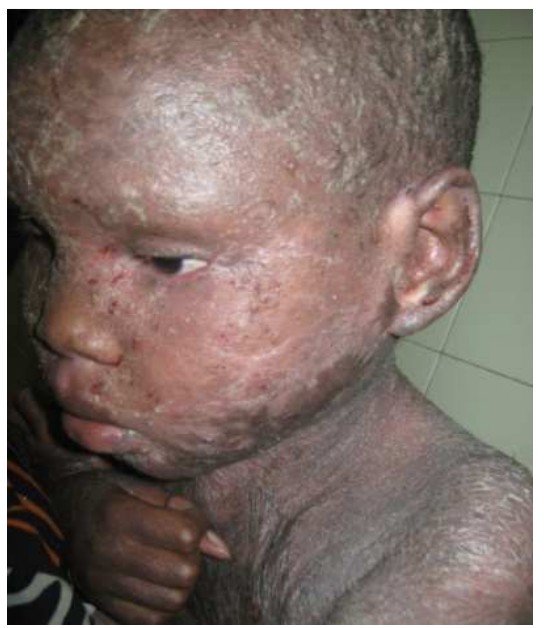

Figure 3. érythrodermic psoriasis.

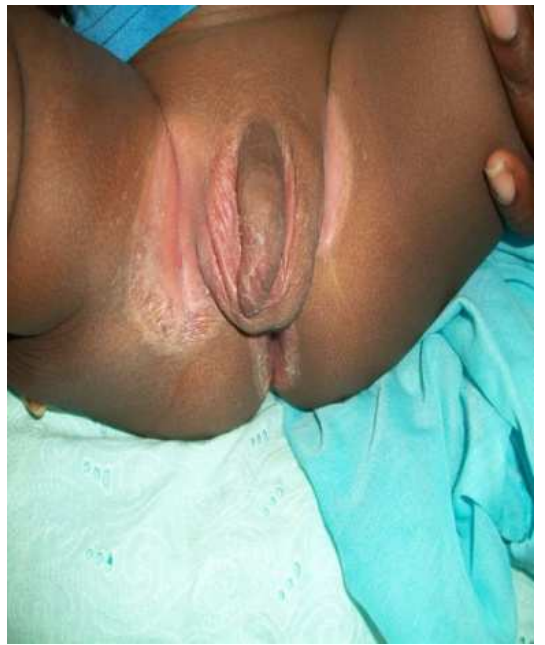

Figure 4 . inverted psoriasis. 


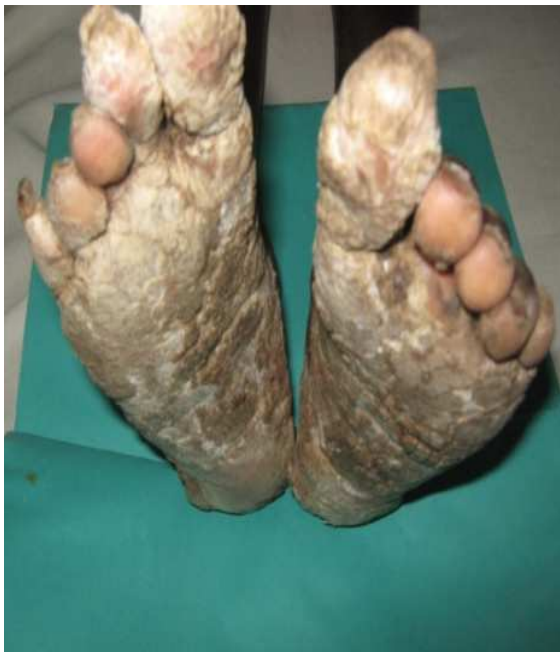

Figure 5. plantar psoriasis.

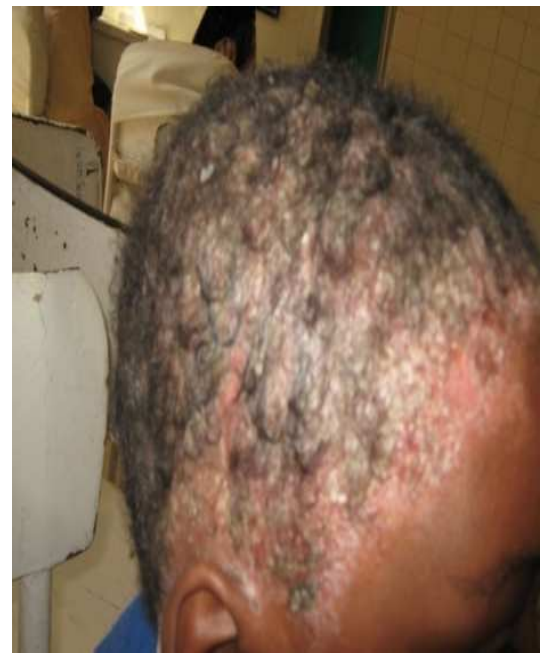

Figure 6. scalp psoriasis.

\section{Discussion}

Psoriasis is a rare disease in childhood. As in the study of Pitche [3] conducted in Togo, which had found 11 cases over a period of 10 years. In our study, children psoriasis accounted for only $0.5 \%$ of pediatric dermatological consultations and $0.67 \%$ of all patients seen in the department during the same period. Children accounted for only $19.4 \%$ of all patients with psoriasis. In a study by Ly et al [4] in the same department relating to 295 patients, psoriasis of the child was only $6 \%$. There was no sex predominance. Kumar's [5], Morris's [6] and Pitche's [3] studies found similar rates of $1.09,1.14$ and 1.15. In most European series, female predominance was clear before 10 years $[7,8]$. In our study, age groups [0-4] and [5-10] were the most representative. We did not find any congenital form. Indeed it is a very rare form [9]. In our study the average age was 6 years. This result is lower than that found by the Pitche in Togo [3] which was 8 years. The absence of pain reflects a part of the long period of consultation. On the other hand the lack of qualified personnel, still testifies diagnostic delay with inadequate and inappropriate prescriptions reflecting ignorance of psoriasis. Atopy was observed in 4 cases (3 asthma and one atopic dermatitis). According to Beer, atopic dermatitis is a predisposing factor psoriatic precede it in half the cases $[10,11]$. This may partly explain the pruritus noted in a third of our patients. The study of Ly et al [4], noted it in half of the patients and had discussed the role of African herbal medicine in the occurrence of pruritus. In addition, scraping is a disseminating factor through the Köbner phenomenon. Clinically psoriasis on black skin is characterized by redness and very thick squames, making diagnosis easier. In our series, the majority was represented by vulgar form $(50 \%)$. This result is similar to Kumar [5] and Seyhan [12] with respective rates of $60 \%$ and $54 \%$. The gutata form, most classic in childhood accounted for $29 \%$ of cases. Seyhan [11] found a similar rate. It is closely related to the existence of a streptococcal infection. In our study antibiotics allowed the regression of lesions. Macrolides were preferred to betalactam antibiotics due to the aggravating effect of these on psoriasis [13]. Elective topography was at the folds in $20 \%$. In its limited form, it poses real problems of differential diagnoses with fungal intertrigo and folds eczema in children. Pustular and erythrodermic psoriasis represented severe forms, facilitated by African herbal medicine of unspecified nature. These may exacerbate dermatitis such as psoriasis [14] and stopping these plants has promoted regression of lesions without creating a systemic treatment. Local treatment has allowed a favorable evolution for $62 \%$ of patient. Therapeutic breaks induced by socio-economic difficulties and the chronic nature of the disease could explain the number of lost sight.

\section{Conclusion}

Psoriasis is a rare condition in children with a predominance of simple forms such as the vulgaris one. Pruritus was frequent, observed in a third of patients. Prognosis was good in most cases, justifying non-aggressive therapeutics.

\section{References}

[1] Ammar M, Zaraa I, Bouchleka S.C, Dhaoui A, Doss N, Ben Osman et al. Le psoriasis familial : étude descriptive à propos de 9 familles. Tunisie médicale, $2009 ; 87: 169$.

[2] Leder RO, Faber EM. The variable incidence of psoriasis in Sub-saharan of Africa. Int J Dermatol. 1997; 36: 911-9.

[3] Pitche P, Agbere A, Kessi K, Tchangai W. Les aspects cliniques du psoriasis de l'enfant au centre hospitalier universitaire de Lomé-Tokoin (Togo). Médecine d'Afrique noire. 1995 ; 42 :305-307.

[4] Ly F, Ndiaye M, Diallo M, Diallo S, DIOP A, Diatta B. A et al. Aspects épidémiologiques, cliniques et thérapeutiques du psoriasis au Sénégal : à propos de 295 cas. Nouv Dermatol 2013; 32 (Suppl. 1): 21-23.

[5] 5. Kumar B, Jain R, Sandhu K. Epidemiology of childhood 
psoriasis: a study of 419 patients from northern India. Int J Dermatol 2004; 43:654-8.

[6] Morris A, Rogers M, Fisher G, Williams K. Childhood psoriasis. A clinical of 1262 cases. Pediatr Dermatol. 2001;18:188-198.

[7] Mahé E, Prost Y. Psoriasis de l'enfant. Pediatr Int. $2004 ; 17: 380-386$.

[8] Guillot B, Guilhou JJ. Le psoriasis et son traitement. J Rheumatol. $2002 ; 69: 615-23$.

[9] Janniger CK, Schwarte RA, Musumeci LM, Tedeschi A, Mirona B, Micali G. Infantile psoriasis. Cutis. 2005; 76:173-177.

[10] Beer WE, Smith AE, Kassab JY, Smith S, Rowland CMV.
Concomitance of psoriasis and atopic dermatitis. Dermatology. 1992; 184:265-70.

[11] Aka Boussou R, Maleville J, Taieb A. Le psoriasis de l'enfant : étude rétrospective de 140 observations. Revue internationale de pédiatrie. $1999 ; 295: 27-31$.

[12] Seyhan M, Kandi Coskun B, Saglam H. Psoriasis in childhood and adolescence: evaluation of demographic and clinical features. Pediatr Int. 2006; 48: 525-530.

[13] Herbst RA, Hoch O, Kapp A, Weiss J. Guttate psoriasis triggered by perianal streptococcal dermatis in a four years old boy. J am Acad Dermatol. 1991; 24: 186-194.

[14] Niang SO. Peau et plantes traditionnelles africaines. Progrès en dermato-allergologie. Besançon 2012 ; 377 : 81-94. 